\title{
Research Paper: The Effectiveness of Education Based on Cognitive, Metacognitive, and Behavioral Model on Reducing Meta-worry Belief in Patients With Social Anxiety Disorder
}

\author{
Afsaneh Shahbazirad ${ }^{1}$, Ezatollah Ghadampour ${ }^{1 *}$, Firoozeh Ghazanfari' ${ }^{1}$, Khodamorad Momeni ${ }^{2}$ \\ 1. Department of Psychology, Faculty of Literature and Human Sciences, Lorestan University, Lorestan, Iran. \\ 2. Department of Psychology, Faculty of Social Sciences, Razi University, Kermanshah, Iran.
}

Article info:

Received: 25 Jun. 2016

Accepted: 14 Oct. 2016

Keywords:

Social anxiety disorder, Cognitive, Metacognitive and behavioral model, Meta-worry belief, Developed model ditation: Shahbazirad, A., Ghadampour, E., Ghazanfari F., Momeni Kh. (2017). The Effectiveness of Education Based on Cognitive, Metacognitive, and Behavioral Model on Reducing Meta-worry Belief in Patients With Social Anxiety Disorder. Journal of Practice in Clinical Psychology, 5(1), 55-62. https://doi.org/10.18869/acadpub.jpcp.5.1.55

doit: https://doi.org/10.18869/acadpub.jpcp.5.1.55

* Corresponding Author:

Ezatollah Ghadampour, PhD

Address: Department of Psychology, Faculty of Literature and Human Sciences, Lorestan University, Lorestan, Iran

Tel: +98 (66) 33120097

E-mail: ghadampoure@lu.ac.ir 


\section{Introduction}

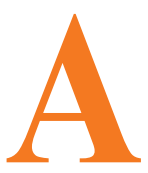

nxiety disorders are the most common psychiatric disorders both in primary care and in clinical medicine. According to previous studies, anxiety disorders cause many complications and problems, such as spending too much time and resources on health care which dramatically reduce patients' quality of life and functions (Sadock \& Sadock, 2007). Social Anxiety Disorder (SAD) is the third most prevalent (13\%) psychiatric disorder (Tulbure, 2011).

Without proper treatment, social anxiety can significantly disturb occupational, educational, and social abilities of patients (Davis, Munson \& Tarcza, 2009). One of the psychological factors involved in social anxiety, is meta-worry (worry about worry) that has drawn much attention in contemporary studies (Wells, 2005). Mellings and Alden in investigating cognitive factors which trigger social anxiety concluded that focusing on oneself and meta-worry are the most important predictors of social anxiety syndromes (Mellings \& Alden, 2000).

Worry associates with catastrophizing which its mental control is difficult. Although worry process is considered as a coping mechanism, the same process can be the center of worry (Borkovec \& Roemer, 1995). If the normal worry turns into pathological worry, metaworry arises. Worry is not only a syndrome of anxiety but also a motivational and active style to evaluate and deal with fear which is particularly important. Apparently, people with anxiety disorders use worry to deal with danger or predictable fear. Meta-worry exacerbates anxiety, however an individual imagines the worry as a defense and does nothing to break the worry chain (Wells, 2000).

In other words, several studies indicate that people with anxiety disorders have more powerful worry beliefs than healthy people (Wells \& Carter, 2001; Ruscio \& Borkovec, 2004). Furthermore, Holeva et al. confirmed the association between anxiety and harmful stress (Holeva, Tarrier \& Wells, 2001). Similarly, the results of periodical studies indicate that using worry to control the thoughts is associated with stress symptoms of students (Roussis \& Wells, 2006).

Different methods have been used to relief meta-worry beliefs. These proven-effective methods comprised cognitive therapy (Boden, John, Goldin, Werner, Heimberg \& Gross, 2012; Wells \& Carter, 2001; Ghahvehchi, Fathi Ashtiani \& Satkin, 2015; Mahjuob \& Teymouri,
2015), metacognitive therapy (Bahadori, Jahanbakhsh, Kajbaf \& Faramarzi, 2012; Salmani, Hasani, MohammadKhani \& Karami, 2014), LEARN multifaceted intervention (Mousavi, Alipour, Zare, Agah Heris \& Janbozorgi, 2014), and attention training technique (Sheykhan, Mohammadkhani \& Hasanabadi, 2013).

However, because of high prevalence of these disorders, their personal, family, and social damages (Wong, Sarver \& Beidel, 2012; Demir, Karacetin, Eralp Demir \& Uysal, 2013), their impact on life quality (Weidman, Fernandez, Levinson, Augustine, Larsen \& Rodebaugh, 2012; Romm, Melle, Thorsen, Andreassen \& Rossberg, 2012), and lack of a comprehensive research on the combination of cognitive, metacognitive, and behavioral factors in the treatment of SAD, it seems that a treatment model, which considers surface structures as well as underlying structure of anxiousness is extremely important.

We believe that in the proposed model, cognition is the starting point in the problem, but interaction with metacognition and behavior is necessary to worsen the problem. In other words, this factors are interrelated. Review of prior research indicates that most proposed models were unable to explain this disorder, so we developed an intervention in this study to rectify these defects. In this regard, initially a model was proposed considering dimensions of cognition (fear of negative evaluation, underlying assumptions, and efficacy), metacognition (mind control, negative meta-beliefs, and cognition ability), and behavior (safety behavior and avoidance behavior). These dimensions were selected because of their important roles in development of SAD. In this regard, a model was designed by considering the theoretical fundamentals of the proposed model, then the effectiveness of education based on cognitive, metacognitive, and behavioral developed model was assessed in reducing meta-worry beliefs in patients with SAD living in Kermanshah City, Iran.

\section{Methods}

This study was a quasi-experimental study with pretest-posttest design and a control group. This randomized clinical trial was conducted at the private clinic of Shekofa, in Kermanshah. The study was conducted from February to April 2016. The statistical population comprised all patients with SAD who referred to private and public consultancy centers in Kermanshah in 2015. The study sample were selected by convenience sampling method. For the present study, a total of 30 patients, who have participated in the structured clinical interview for DSM-IV Axis I Disorders (SCID-I) and 
diagnosed as having SAD were selected and randomly assigned in two groups of experiment and control. The information was collected to assess the effectiveness of education based on cognitive, metacognitive, and behavioral model (independent variable) on the reduction of meta-worry belief (dependent variable) among the patients with SAD in Kermanshah, Iran.

The study inclusion criteria were as follows; two standard deviations higher than the subscale of meta-worry, diagnosis of social anxiety based on SCID-I, the age range of 20-40 years, and under no psychiatric medication.

The study exclusion criteria were as follows: other physical and mental diseases such as personality disorder, depression disorder, bipolar disorder as main diagnosis (because of potential impact of these disorders on dependent variables), use of psychotic drugs, absence in the pretest and posttest. In this study, the following ethical aspects were observed: The study participants had the discretion to cooperate and their consent was taken orally. The information of participants was kept confidential and were reported in groups. After the final therapy session, several therapeutic sessions were held for the control group.
The following instruments were used to collect the data; Structured clinical interview for axis 1 disorders (SCID-I), SCID-I is applicable for axis 1 disorders and has two versions: clinical version (SCID-CV) and research version. In this study, the clinical version was used. This version covers most common disorders in clinics. It is shorter than the research version and starts with an open interview about the current disease and the previous periods of disease (Mohamamdkhani, 2010). Sharifi et al. (1999) found interrater agreement of diagnosis of the instrument as moderate to good (kappa coefficient more than 60.0). They have also estimated the overall kappa of the current diagnosis as 52.0 and the overall kappa of lifetime diagnosis as 55.0 (Hasani, Tajodini, Ghaedniyaie-Jahromi \& Farmani-Shahreza, 2014). Agreement kappa index was estimated for the categorical diagnosis as 48.0 to 98.8 and the interclass diagnosis as 90.0 to 98.0 (Maffei, Fossati, Agostoni, Donati, Namia, Novella et al., 1997).

Scale of Well's anxious thoughts (1994) is a 21-item tool, 7 items measure meta-worry, 8 items social anxiety, and 6 items health anxiety. In each subscale, which is scored based on the Likert-type scale (4-point), the internal consistency of meta-worry was reported as 0.81 , social anxiety as 0.85 , and health anxiousness as

Table 1. Content of cognitive, metacognitive, and behavioral model in reduction of meta-worry belief

\begin{tabular}{|c|c|}
\hline Sessions & Content \\
\hline First session & $\begin{array}{l}\text { Introduction and communication among group members, familiarity with the framework and the rules of } \\
\text { participation in sessions }\end{array}$ \\
\hline Second session & $\begin{array}{l}\text { Explanation of disorder in the context of biopsychosocial disorders and definition of the signs and symptoms } \\
\text { considered as a part of well-known marks }\end{array}$ \\
\hline Third session & $\begin{array}{l}\text { Recognition of musts and challenges with must-thinking challenges, behavioral experiments, rewards for } \\
\text { individual contracts (works on aspect of cognition) }\end{array}$ \\
\hline Forth session & $\begin{array}{l}\text { Recognition of usefulness of assumptions and rules, listing its costs and benefits, imagery to modify assump- } \\
\text { tions and rules (works on aspect of cognition) }\end{array}$ \\
\hline Fifth session & $\begin{array}{l}\text { Techniques of differentiation of thoughts and reality and techniques of bordering between progress-oriented } \\
\text { and perfectionism (works on aspect of cognition) }\end{array}$ \\
\hline Sixth session & $\begin{array}{l}\text { Educating efficacy and self-tools, identifying negative self-talk and cognitive disorders (works on aspect of } \\
\text { cognition) }\end{array}$ \\
\hline Seventh session & Relaxation training and desensitization (works on aspect of behavior) \\
\hline Eighth session & Training coping skills, self-adjusting, thought stopping, and role playing (works on aspect of behavior) \\
\hline Ninth session & $\begin{array}{l}\text { Introduction of techniques to delay mental rumination, reform one's positive and negative meta-beliefs, at- } \\
\text { tention training technique (works on aspect of metacognition) }\end{array}$ \\
\hline Tenth session & $\begin{array}{l}\text { Evaluation and identification of metacognitive control strategies and substituting useful metacognitive } \\
\text { control strategies, techniques of getting away from mindfulness, dealing with strategies and metacognitive } \\
\text { dysfunctions (works on aspect of metacognition) }\end{array}$ \\
\hline Eleventh session & $\begin{array}{l}\text { Integration of cognitive, metacognitive, and behavioral techniques in practice (works on aspects of cognition, } \\
\text { metacognition, and behavior) }\end{array}$ \\
\hline Twelfth session & Sum up and review homework \\
\hline
\end{tabular}


0.74 . The reliability of this scale was 0.92 with test-retest method and 0.89 with the split-half method (Fata, Mootabi, Moloodi \& Ziayee, 2010). In this research, meta-worry subscale was used and the reliability of this scale was 0.97 through Chronbach's alpha.

After selecting and randomly assigning the patients with SAD in two groups of experiment and control, the patients in the experimental group received education by cognitive, metacognitive, and behavioral model designed based on the theory of Neenan and Dryden (1953), Leahy (2003), Beck (1976), Wells (1962), and Bandura (1997). This educational program was administered by the researcher in two months (two 90-minute sessions per week. Since the selected patients were literate the questionnaires were filled by the patients themselves (Table 1). During the implementation of the intervention program for the experiment group, the control group received no intervention. After the sessions, both groups were evaluated by meta-worry belief subscale of Well's questionnaire of anxious thoughts (1994). The obtained data from the questionnaire were analyzed by analysis of covariance (ANCOVA) through using SPSS (Version 19).

\section{Results}

All participants were in the age range of 20-40 years. The average age of patients was $29.38 \pm 5$ years and all of them had the academic education. Also both experiment group and control group were matched with regard to demographic characteristics.
Figure 1 shows that the average meta-worry belief in the experiment group decreased in the posttest (10.08) compared to pretest (19.6). However, average metaworry belief in the control group in the posttest (19.4) compared to pretest (20.19) did not show any significant reduction (Table 2).

Before the covariance analysis, the assumption of normality and homogeneity of variances were assessed. For normality, skewness and kurtosis were used and since the score of meta-worry belief ranged $0.797-0$. 261 in the pretest and $0.02-1.7$ in the posttest (within the normal range $[-2,+2])$, it has the normal distribution. Levene's test was used for equality of variances' assumptions. Due to the absence of meaningful levels $(0.5,0.8)$ in pretest and posttest, the homogeneity of variances can be established about meta-worry variable.

After adjusting the pretest scores, the effectiveness of cognitive, metacognitive, and behavioral model on the reduction of meta-worry belief in patients with $\mathrm{SAD}$ was evaluated. Obtained value of $\mathrm{F}=346.9$ in the meaningful level of $\mathrm{P}<0.001$, demonstrated the effectiveness of cognitive, metacognitive, and behavioral model on the reduction of meta-worry belief. Considering the evaluated amount of eta and its meaningfulness, $92 \%$ of meta-worry belief variance is explained by independent variable of educating cognitive, metacognitive, and behavioral model (Table 3).

\section{Discussion}

This study was conducted to investigate the effectiveness of educating cognitive, meta-cognitive, and behav-

Table 2. Descriptive indicators for meta-worry belief

\begin{tabular}{cccccc}
\hline & \multicolumn{3}{c}{ Meta-Worry Belief } & \\
\hline & Groups & & \multicolumn{2}{c}{ Experimental } & Control \\
\hline Phase & Mean & SD & Mean & SD \\
\hline Pretest & 19.6 & 1.6 & 20.19 & 1.7 \\
Posttest & 10.08 & 1.4 & 19.4 & 1.3 \\
\hline
\end{tabular}

PRACTIE in
CLINICAL PSYCH $\cong$ LOGY

Table 3. Analysis of ANCOVA for meta-worry belief

\begin{tabular}{ccccccc}
\hline Statistical Descriptive & Mean Square & df & Sum of Squares & F & Sig. & Eta Squared \\
\hline Pretest & 7.63 & 1 & 7.63 & 4.24 & 0.04 & 0.136 \\
Group & 624.01 & 1 & 624.01 & 346.9 & 0.001 & 0.928 \\
\hline
\end{tabular}




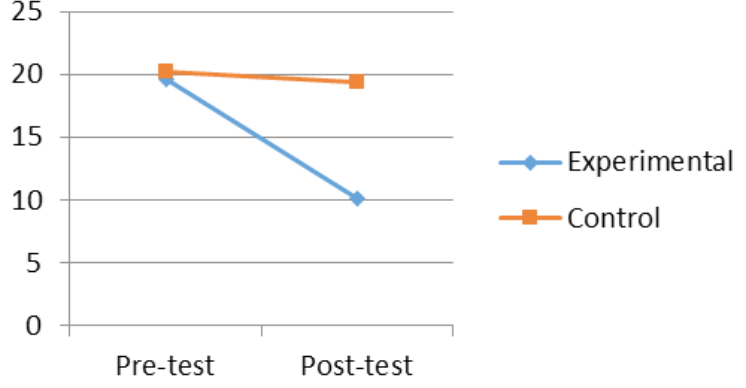

PRACTICE in CLINICAL PSYH $\mathbb{Q}$ LOGY

Figure 1. Comparison of average meta-worry belief in pretest and posttest in experiment and control groups

ioral developed model on the reduction of meta-worry belief in patients with SAD in Kermanshah, Iran. The results of the present study showed that educating the developed model had a meaningful impact on the reduction of meta-worry belief. Because there was not any study on combined aspects of cognition, metacognition and behavioral, therefore similar research was mentioned in explaining the results. Similar research in this area include Boden et al. (2012), Wells and Carter (2001), Mahjuob and Teymouri (2015), Ghahvehchi et al. (2015), Bahadori et al. (2012), and Salmani et al. (2014).

Patients with SAD know that dangerous and uncontrollable thoughts are associated with frightening situations. They sometimes consider such thoughts positively, because it allows them to preserve themselves in dangers. This reaction leads to a lot of rumination and rising their awareness of relevant thoughts to social anxiety. However, in this treatment approach, the patients try to control and change metacognition responsible for increasing the style of maladaptive thoughts or negative beliefs. In this approach, such techniques and methods are taught that are used against intrusive thoughts about social anxiety.

The combined model affects negative meta-worry beliefs about uncontrollability and dangerousness of worry in a way that the behaviors like seeking or avoiding certain clues causing worry would change and it is possible to find the opportunities of learning controllability and harmlessness of worry. On one hand, it suppresses thoughts, which lead to more unwanted thoughts and controllability of worry and provides the opportunity for anxious people to use re-evaluation as a positive and constructive strategy.

On the other hand, considering that the intervention was a combination of cognitive techniques such as guided self-talk, moderate assumptions and attitudes, cognitive distortion correction; behavioral techniques like relaxation of tensions, coping skills; and metacognitive skills such as correction of positive and negative meta-beliefs, and replacement of useful metacognitive strategies, education as a technique of attention, can affect directly and indirectly cognitive, metacognitive, and behavioral aspects, and accordingly reduce metaworry beliefs in patients.

Furthermore, the patients by considering meta-cognitive approach beside the cognitive and behavioral approach, learn a kind of information process in addition to their deep and superficial beliefs and attitudes to analyze information in a way that the activity stimulator not be insufficient and self-adjusting model and this causes the reduction of meta-worry belief in patients.

Cognition, metacognition, and behavioral intervention enable patients to have positive beliefs about themselves and their surrounding events, and reassess their reactions. They also try to get a realistic impression about problems and subjects. However, more research is necessary on the effectiveness of this model and comparing it with other treatment methods. In general, it seems that application of this technique is clinically effective and costs less for patients. By teaching techniques of this model to patients with social anxiety, they spend less on medications.

In the end, it is worth noting that this research demands more study considering the generalization of its results. Due to the limited sample of patients in Kermanshah and also self-reported instruments, we should be cautious in generalizing the results. The other limitations were the intervention program which was handled by the researcher that could affect the results orientation, lack of evaluation of biological, social and personality factors in this research, no control over variables like personality and mental states of patients, no comparison between mentioned model and other treatments, and other aspects of cognition, metacognition and behavior were missed in this study because of extension of study.

In the future research, the effectiveness of this treatment method could be examined on other social anxiety disorders (phobia, generalized anxiety). The model could also be compared with other therapies until usefulness of this model be investigated in the long term. Since in this study, group method was used for education theory, it is recommended that the individual method be used in future studies. Efficacy of treatment is suggested to be studied with larger samples, longer periods, and follow-up. In future studies, other aspects 
of cognition, metacognition and behavior could be studied, too.

Although previous studies approved the effectiveness of cognition-behavioral and meta-cognition treatment alone on anxiety, but it should be noted that each of these treatments affect some parts of one's personality, while our treatment combined three different aspects and tried to affect cognition, metacognition and behavioral aspects to create stable effects. Thus, the results of this study have important implications in the treatment and counseling services for patients with SAD, for example, therapists and counselors can use this combination model to reduce meta-worry belief in patients with SAD.

\section{Acknowledgments}

The authors would like to acknowledge all people who participated in the study. This article was extracted from the $\mathrm{PhD}$ thesis of the first author in Psychology. The authors received no financial support for the research, authorship and/or publication of this article. Afsaneh Shahbazirad contributed in the conception of the work and conducting the study. Ezatollah Ghadampour was the advisor. Firoozeh Ghazanfari and Khodamorad Momeni were consulting advisors in conducting the study.

\section{Conflict of Interest}

The authors declared no conflict of interests.

\section{References}

Bahadori, M. H., Jahanbakhsh, M., Kajbaf, M. B., \& Faramarzi, S. (2012). [The effectiveness of meta-cognitive therapy on meta-cognitive beliefs and cognitive trust in patients with social phobia disorder (Persian)]. Journal of Clinical Psychology, 4(1), 33-41.

Bandura, A. (1997). Self-efficacy: Toward a unifying theory of behavioral change. Psychological Review, 84(2), 191-215. doi: 10.1037//0033-295x.84.2.191

Beck, A. T. (1976). Cognitive therapy and emotional disorders. New York: International University Press.

Boden, M. T., John, O. P., Goldin, P. R., Werner, K., Heimberg, R. H., \& Gross, J. J. (2012). The role of maladaptive beliefs in cognitive-behavioral therapy: Evidence from social anxiety disorder. Behavior Research \& Therapy, 50(5), 287-291. doi: 10.1016/j.brat.2012.02.007

Borkovec, T. D., \& Roemer, L. (1995). Perceived functions of worry among generalized anxiety subjects: Distraction from more emotionally distressing topics? Journal of Behav- ior Therapy and Experimental Psychiatry, 26(1), 25-30. doi: 10.1016/0005-7916(94)00064-s

Davis, T. E., Munson, M. S., \& Tarcza, E. V. (2009). Anxiety disorders and phobias. In J. L. Matson (Ed.), Social Behavior and Skills in Children (pp. 219-243). New York: Springer.

Demir, T., Karacetin, G., Eralp Demir, D., \& Uysal, O. (2013). Prevalence and some psychosocial characteristics of social anxiety disorder in an urban population of Turkish children and adolescents. European Psychiatry, 28(1), 64-69. doi: 10.1016/j.eurpsy.2011.12.003

Fata, L., Mootabi, F., Moloodi, R., \& Ziayee, K. (2010). [Psychometric properties of Persian version of thought control questionnaire and anxious thought inventory in Iranian students (Persian)]. Journal of Psychological Models and Methods, 1(1), 33-45.

Ghahvechi Hosseini, F., Fathi Ashtiani, A., \& Satkin, M. (2015). Comparing meta-cognitive therapy with cognitive therapy on reducing test anxiety and meta-worry in students. Practice in Clinical Psychology, 3(3), 213-219.

Hasani, J., Tajodini, E., Ghaedniyaie-Jahromi, A., FarmaniShahreza, Sh. (2014). [The assessments of cognitive emotion regulation strategies and emotional schemas in spouses of people with substance abuse and spouses of normal people (Persian)]. Journal of Clinical Psychology, 1(21), 91-101.

Holeva, V., Tarrier, N., \& Wells, A. (2001). Prevalence and predictors of acute PTSD following road traffic accident: Thought control strategies and social support. Behavior Therapy, 32(1), 65-83. doi: 10.1016/ s0005-7894(01)80044-7

Leahy, R. L. (2003). Cognitive therapy techniques: A practitioners guide, New York: Guilford Press.

Maffei, C., Fossati, A., Agostoni, I., Donati, D., Namia, C., Novella, L., \& Petrachi, M. (1997). Interrater reliability and internal consistency of the structured clinical interview for DSM-IV Axis II personality disorders (SCID-II), version 2.0. Journal of Personality Disorder, 11(3), 279-284. doi: 10.1521/ pedi.1997.11.3.279

Mahjuob, N., \& Teymouri, S. (2015). [The effectiveness of mindfulness-based cognitive therapy on reducing anxiety sensitivity and meta-worry in students with social anxiety (Persian)]. Journal of Clinical Psychology, 7(2), 39-48.

Mellings, T. M., \& Alden, L. E. (2000). Cognitive processes in social anxiety: The effects of self-focus, rumination and anticipatory processing. Behavior Research and Therapy, 38(3), 243-257. doi: 10.1016/s0005-7967(99)00040-6

Mohamamdkhani, P. (2010). [Structured clinical interview for disorders DSM-IV-TR 9 (Persian)]. Tehran: Danjeh.

Mousavi, E., Alipour, A., Zare, H., Agah Heris, M., \& Janbozorgi, M. (2014). Effectiveness of learn multifaceted intervention in modifying metacognition and the meta-worry beliefs. Advanced Cognition Science, 16(1), 39-48.

Neenan, M., \& Dryden, W. (1953). Cognitive theory: 100 key point and techniques, New York: Rutledge.

Romm, K. L., Melle, I., Thorsen, C., Andreassen, O. A., \& Rossberg, J. I. (2012). Severe social anxiety in early psychosis in associated with poor premorbid functioning, depression, and reduced quality of life. Compare Psychiatry, 53(5), 434440. doi: 10.1016/j.comppsych.2011.06.002 
Roussis, P., \& Wells, A. (2006). Post-traumatic stress symptoms: tests of relationships with thought control strategies and beliefs as predicted by the meta cognitive model. Personality Individual Difference, 40(1), 111-122. doi: 10.1016/j. paid.2005.06.019

Ruscio, A. M., \& Borkovec, T. D. (2004). Experience and appraisal of worry among high worriers with and without generalized anxiety disorder. Behavior Research and Therapy, 42(12), 1469-82. doi: 10.1016/j.brat.2003.10.007

Sadock, H. S., \& Sadock, B. J. (2007). Synopsis of psychiatry: behavioral Sciences/Clinical Psychiatry (10 ${ }^{\text {th }}$ ed.). Philadelphia: Lippincott Willimas \& Wiilkins.

Salmani, B., Hasani, J., Mohammad-Khani, Sh., \& Karami, G. H. R. (2014). [The efficacy of metacognitive therapy on metacognitive beliefs, metaworry and the signs and symptoms of patients with generalized anxiety disorder (Persian)]. Feyz, 18(5), 428-439.

Sheykhan, R., Mohammadkhani, Sh., \& Hasanabadi, H. R. (2013). The effect of the group training of the attention training technique on anxiety, self-focused attention and metaworries in socially anxious adolescents. Journal of Cognitive Psychology, 1(1), 33-45.

Tulbure, B. T. (2011). The efficacy of Internet supported intervention for social anxiety disorder: A brief meta-analytic review. Journal of Social Behavior Science, 30, 552-57. doi: 10.1016/j.sbspro.2011.10.108

Weidman, A. C., Fernandez, K. C., Levinson, C. A., Augustine, A. A., Larsen, R. J., \& Rodebaugh, T. L. (2012). Compensatory internet use among individuals higher in social anxiety and its implications for well-being. Journal of Personality Individual Difference, 53(3), 191-195. doi: 10.1016/j. paid.2012.03.003

Wells, A. (1962). Meta cognitive therapy for anxiety and depression. New York: Guilford.

Wells, A. (2000). Emotional disorders and meta cognition: innovative cognitive therapy. Chichester, UK: John Wiley \& Sons.

Wells, A. (2005). The meta cognitive model of GAD: Assessment of met worry and relationship with DSM-IV generalized anxiety disorder. Cognitive Therapy Research, 29(1), 107-121. doi: 10.1007/s10608-005-1652-0

Wells, A., \& Carter, K. (2001). Further tests of a cognitive model of Generalized Anxiety Disorder: Meta cognitions and worry in GAD, panic disorder, social phobia, depression, and non-patients. Journal of Behavior Therapy, 32(1), 85-102. doi: 10.1016/s0005-7894(01)80045-9

Wong, N., Sarver, D. E., \& Beidel, D. C. (2012). Quality of life impairments among adults with social phobia: The impact of subtype. Journal of Anxiety Disorder, 26(1), 50-57. doi: 10.1016/j.janxdis.2011.08.012 
